\title{
Formulation and Evaluation of Mucoadhesive Microspheres of Pioglitazone Hydrochloride Prepared by Ionotropic External Gelation Technique
}

\author{
Nagarajan Sriram1, Prakash Katakam ${ }^{2}$ \\ ${ }^{1}$ Department of Biotechnology, Acharya Nagarjuna University, Guntur, India \\ ${ }^{2}$ Department of Pharmaceutics, Priyadarshini Institute of Pharmaceutical Education and Research, \\ Guntur, India \\ Email: "pkatakam9@gmail.com
}

Received 15 December 2015; accepted 12 March 2016; published 15 March 2016

Copyright (C) 2016 by authors and Scientific Research Publishing Inc.

This work is licensed under the Creative Commons Attribution International License (CC BY). http://creativecommons.org/licenses/by/4.0/

$$
\text { Open Access }
$$

\section{Abstract}

Microspheres containing Pioglitazone hydrochloride were prepared by the ionotropic external gelation method, using sodium alginate with four mucoadhesive polymers namely sodium carboxy methyl cellulose, hydroxy propyl methyl cellulose, carbopol $934 \mathrm{P}$ and cellulose acetate phthalate as coat materials. Ionotropic gelation is a method to prepare microspheres using combination of $\mathrm{Ca}^{2+}$ as cationic components and alginate as anion. The practical yield of prepared microspheres using the ionotropic gelation technique was between $172 \mathrm{mg}$ and $604 \mathrm{mg}$. The result of the Chisquared test carried out between the actual (practical) and expected (theoretical) yields showed no significant difference $(P<0.05)$ which indicated that the ionotropic gelation technique could be successfully employed to prepare pioglitazone microspheres using sodium alginate, sodium carboxy methyl cellulose, carbopol 934 P, HPMC, cellulose acetate butyrate polymers. The drug entrapment efficiency of prepared microspheres showed between $56.12 \% \pm 3.86 \%$ to $84.68 \% \pm$ $2.93 \%$ which was significantly higher for ionotropic gelation technique. The highest drug entrapment was found in formulation PMI 8. Swelling index is the capability of a polymer to swell before the drug is released which influences the rate and mechanism of drug release from the polymer matrix. The swelling index of prepared microspheres was in the range of $68 \% \pm 4.52 \%$ to $87 \% \pm$ $0.98 \%$. Pioglitazone $\mathrm{HCl}$ microspheres showed controlled release of drug without initial peak level achieving. This type of properties in Pioglitazone $\mathrm{HCl}$ microspheres used to decrease side effects, reduce dosing frequency and improve patient compliances. From the all batches PMI 8 is considered the best formulation, because among all other formulations, it shows better extent of drug

\footnotetext{
${ }^{*}$ Corresponding author.
} 
release up to $82.12 \%(18 \mathrm{~h})$, good entrapment efficiency $(84.68 \%)$ and the ex-vivo wash-off test shows the best mucoadhesive property. The in vitro drug release studies do up to $18 \mathrm{~h}$. As observed from the various plots, most of the formulations followed the Korsmeyer-Peppas model.

\title{
Keywords
}

\author{
Diabetes Mellitus, Mucoadhesive Microspheres, Mucoadhesive Polymers, Mucoadhesion, \\ Sustained Release, Ionotropic External Gelation Technique
}

\section{Introduction}

Microspheres are discrete particles that make up a multiple unit system. Recently, much emphasis has been laid on the development of microspheres dosage forms in preference to single unit systems because of their potential benefits such as increased bioavailability, reduced risk of systemic toxicity, reduced risk of local irritation and predictable gastric emptying. Microspheres systems show better reproducible pharmacokinetic behavior than conventional (monolithic) formulations. The incorporation of mucoadhesive polymers in the microspheres significantly increases the gastrointestinal transit time of microspheres [1]. It has the desired characteristics suitable for developing mucoadhesive extended release formulations, which include its solubility in acidic $\mathrm{pH}$ and a shorter half-life of $3-5 \mathrm{~h}$. Due to side effects of pioglitazone $\mathrm{HCl}$ a sustained release medication is required to get prolonged effect with reduced fluctuations in drug plasma concentration levels. Mucoadhesive microspheres can provide the sustained release of drugs and advantage for pioglitazone $\mathrm{HCl}$ in the management of type-2 diabetes with a high margin of safety and reduced side effects.

The literature survey revealed scanty research on mucoadhesive microspheres of pioglitazone, which were prepared by using a solvent evaporation method using ethylcellulose, HPMC, and eudragit [2]. Hence the present investigation has been carried out to prepare and evaluate mucoadhesive microspheres of pioglitazone $\mathrm{HCl}$ employing ionotropic external gelation method using sodium alginate in combination with four mucoadhesive polymers namely sodium carboxy methyl cellulose, carbopol $934 \mathrm{P}$, hydroxy propyl methyl cellulose, and cellulose acetate phthalate as coat materials.

\section{Materials and Methods}

\subsection{Materials}

Pioglitazone hydrochloride a gift sample obtained from Torrent Pharmaceuticals, Ahmadabad, sodium alginate obtained from Loba chemie, mumbai, hydroxy propyl methyl cellulose, carbopol $934 \mathrm{P}$, cellulose acetate Phthalate from Glenmark Pharmaceuticals, and all chemicals used are of analytical grade.

\subsection{Preparation of Microspheres}

Microspheres containing Pioglitazone hydrochloride hydrochloride were prepared by the ionotropic external deletion method, using sodium alginate in combination with four mucoadhesive polymers namely hydroxy propyl methyl cellulose, carbopol $934 \mathrm{P}$, and cellulose acetate phthalate (Table 1). Ionotropic gelation is a method to prepare microspheres using combination of $\mathrm{Ca}^{2+}$ as cationic components and alginate as anion [3]. In this method the $5 \% \mathrm{w} / \mathrm{v}$ sodium alginate solution as prepared dispersing the weighed amount of sodium alginate with weighed quantity of mucoadhesive polymer in deionized water and stirred with for $2 \mathrm{~h}$ using a magnetic stirrer. In between the time of stirring, add accurately weighed quantity of Pioglitazone hydrochloride $\mathrm{HCl}$, which is dissolved in methanol and stirring continued with gentle heat to get a homogenous drug-polymer mixture. For the formation of microspheres, drug-polymer solution was extruded drop wise from a needle of $18 \mathrm{G}$ in diameter from a height of about $6 \mathrm{~cm}$ into $100 \mathrm{ml}$ aqueous $2 \%$ calcium chloride solution and stirred at 100 RPM. Then the solution containing the gel formed microspheres was filtered by using Whitman filter paper No-1. The added droplets were kept dispersed in the calcium chloride solution for 15 minutes to complete the curing reactions and to produce spherical rigid microspheres. The microspheres were collected by decantation, and the product, thus separated, was washed repeatedly with water. The microspheres were allowed to dry at about $30^{\circ} \mathrm{C}-$ 
Table 1. Formulation of pioglitazone microspheres.

\begin{tabular}{cccc}
\hline S. No & Formulation code & Coating polymer composition & Ratio \\
\hline 1 & PMI 1 & Drug: Sodium Alginate & $1: 1$ \\
2 & PMI 2 & Drug: Sodium Alginate & $1: 2$ \\
3 & PMI 3 & Drug: Sodium Alginate & $1: 3$ \\
4 & PMI 4 & Drug: Sodium Alginate: SCMC & $1: 1: 1$ \\
5 & PMI 5 & Drug: Sodium Alginate: SCMC & $1: 1: 2$ \\
6 & PMI 6 & Drug: Sodium Alginate: SCMC & $1: 1: 3$ \\
7 & PMI 7 & Drug: Sodium Alginate: Carbopol 934 P & $1: 1: 1$ \\
8 & PMI 8 & Drug: Sodium Alginate: Carbopol 934 P & $1: 1: 2$ \\
9 & PMI 9 & Drug: Sodium Alginate: Carbopol 934 P & $1: 1: 3$ \\
10 & PMI 10 & Drug: Sodium Alginate: HPMC & $1: 1: 1$ \\
11 & PMI 11 & Drug: Sodium Alginate: HPMC & $1: 1: 2$ \\
12 & PMI 12 & Drug: Sodium Alginate: HPMC & $1: 1: 3$ \\
13 & PMI 13 & Drug: Sodium Alginate: CAB & $1: 1: 1$ \\
14 & PMI 14 & Drug: Sodium Alginate: CAB & $1: 1: 2$ \\
15 & PMI 15 & Drug: Sodium Alginate: CAB & $1: 1: 3$ \\
\hline
\end{tabular}

SCMC-Sodium carboxy methyl cellulose; CAB-Cellulose Acetate Butyrate; HPMC-Hydroxy Propyl Methyl Cellulose.

$40^{\circ} \mathrm{C}$ for $12-48 \mathrm{~h}$, and stored in well closed container for further use.

\section{Preformulation Studies}

\subsection{FTIR Spectroscopy [4]}

Spectral analysis of pure drug and physical mixture of drug and different excipients which were used for preparation of microspheres was studied by FTIR. Potassium bromide $(\mathrm{KBr})$ disks were prepared by mixing $10 \mathrm{mg}$ of sample with $\mathrm{KBr}$ by compacting in a hydrostatic press under vacuum at $6-8$ tons pressure. The resulting disc was mounted on a suitable holder in IR spectrophotometer and the spectrum was recorded from $4000 \mathrm{~cm}^{-1}$ to $400 \mathrm{~cm}^{-1}$ at a scan time of $12 \mathrm{~min}$. The resultant spectrum was compared for any spectral changes. They were observed in the presence of characteristic peaks in the respective functional groups in the sample compounds.

\subsection{Differential Scanning Calorimetry [5]}

The differential scanning calorimetry (DSC) analysis of pure pioglitazone $\mathrm{HCl}$, blank microspheres and drug loaded microspheres. The DSC analysis was conducted in the heating range from $40^{\circ} \mathrm{C}$ to $300^{\circ} \mathrm{C}$ at a rate of $10^{\circ} \mathrm{C} \cdot \mathrm{min}^{-1}$ using TAG 1000 differential scanning calorimeter.

\section{Evaluation of Formulated Microspheres}

\subsection{Percentage Yield [6]}

The prepared and dried microspheres of all batches were accurately weighed. The measured weight of prepared microspheres was divided by the total amount of all the excipients and drug used in the preparation of the microspheres, which give the total percentage yield of microspheres (Table 2). It was calculated using the following equation

$$
\text { Percentage yield }=\frac{\text { Weight of microspheres }}{\text { Weight of the polymer }+ \text { weight of pioglitazone } \mathrm{HCl}} \times 100 \text {. }
$$

\subsection{Drug Content and Entrapment Efficiency [7] [8]}

The formulated microspheres of pioglitazone $\mathrm{HCl}$ were taken in a mortar and crushed. Then the microspheres 
Table 2. Percentage yield of Pioglitazone microspheres.

\begin{tabular}{cccc}
\hline Formulation code & Theoretical weight $(\mathbf{m g})$ & Practical yield $(\mathbf{m g})$ & Percentage yield (\%) \\
\hline PMI 1 & 300 & 172 & 57.33 \\
PMI 2 & 450 & 253 & 56.22 \\
PMI 3 & 600 & 298 & 49.66 \\
PMI 4 & 450 & 332 & 73.77 \\
PMI 5 & 600 & 452 & 75.33 \\
PMI 6 & 750 & 591 & 78.88 \\
PMI 7 & 450 & 379 & 84.22 \\
PMI 8 & 600 & 526 & 87.66 \\
PMI 9 & 750 & 604 & 80.53 \\
PMI 10 & 450 & 358 & 79.55 \\
PMI 11 & 600 & 422 & 70.33 \\
PMI 12 & 750 & 548 & 73.06 \\
PMI 13 & 450 & 371 & 82.44 \\
PMI 14 & 600 & 463 & 77.16 \\
PMI 15 & 750 & 532 & 70.93 \\
\hline
\end{tabular}

Mean \pm standard deviation $(\mathrm{n}=3)$.

were dissolved in $5 \mathrm{ml}$ of methanol and drug was extracted into aliquots of $0.1 \mathrm{~N} \mathrm{HCl}(\mathrm{pH} 1.2)$ repeatedly by evaporating methanol. The extract was transferred to a $100 \mathrm{ml}$ volumetric flask and the volume was made up to $100 \mathrm{ml}$ using $0.1 \mathrm{~N} \mathrm{HCl}$. The solution was kept aside for $24 \mathrm{~h}$ to equilibrate at room temperature. The solution was filtered through $0.45 \mu$ membrane filter paper, diluted suitably and the absorbance was analyzed spectrophotometrically (Shimadzu UV 1700) at $269 \mathrm{~nm}$. The amount of drug entrapped in the microspheres was calculated using the following equations.

$$
\begin{gathered}
\text { Percentage of drug loading }=\frac{\text { Amount of the drug in microspheres }}{\text { Total weight of microspheres }} \times 100 \\
\text { Percentage of drug entrapment }=\frac{\% \text { of drug loading }}{\% \text { of theoretical drug loading }} \times 100
\end{gathered}
$$

\subsection{Morphological Study Using SEM}

The external morphological study was carried out by scanning electron microscope (SEM) (HITACHI SU 3700, Japan) operating at $15 \mathrm{kv}$. The optimized microspheres were taken for the surface characteristic studies. The samples were mounted on a metal stub with double adhesive tape and coated with a sputter under vacuum. The shape and surface characteristic of the microspheres was observed in photographs [9].

\subsection{Size of the Microspheres [10]}

Size distribution plays a very important role in determining the release characteristics of the microspheres. The size distributions in terms of average diameter of the microspheres were determined by the optical microscope method. Microspheres have a size range of $1-1000 \mu \mathrm{m}$. A minute quantity of dried microspheres was suspended in glycerin and the particle size of 100 microspheres was determined in each batch and final the particle size was calculated. In this method, a compound microscope fitted with a calibrated ocular micrometer and a stage micrometer slide was used to count the microspheres.

\subsection{Swelling Index [11]}

The swelling index of microspheres was evaluated by a known weight $(100 \mathrm{mg})$ of drug loaded microspheres 
which were placed in $100 \mathrm{ml}$ of simulated intestinal fluid (SIF, pH 7.4 phosphate buffer) and allowed to swell for the required period of time. The excess surface adhered liquid drops were removed by blotting with filter paper and swollen microspheres were weighed using microbalance. The swelling index was calculated from the difference between the initial weight of the microspheres and the weight at the time of determination using the formula:

$$
\% \text { Swelling index }=\frac{\mathrm{Wf}-\mathrm{Wi}}{\mathrm{Wi}} \times 100
$$

where, $\mathrm{Wi}$ and $\mathrm{Wf}$ are the initial and final weights of microspheres.

\subsection{Ex-Vivo Mucoadhesion Study [12] [13]}

The mucoadhesive property of the microspheres was studied using ex-vivo mucoadhesion method. In this method, freshly excised piece of goat intestinal mucosa $(2 \times 3 \mathrm{~cm})$ was mounted onto glass slides with elastic bands. About 100 microspheres were spread onto the wet rinsed intestinal mucosa and there after the support was hung onto the arm of a USP tablet disintegrating test machine. The disintegration machine containing tissue specimen was adjusted for a slow and regular up and down moment in a test fluid at $37^{\circ} \mathrm{C}$ taken in a beaker. At the end of $1 \mathrm{~h}$ and later at hourly intervals up to $8 \mathrm{~h}$, the machine was stopped and the number of microspheres still adhering onto the tissue was counted and the percentage of mucoadhesion was calculated. The test was performed in $\mathrm{pH} 1.2 \mathrm{HCl}$ buffer and $\mathrm{pH} 7.4$ phosphate buffer.

\subsection{In-Vitro Dissolution Studies}

The in-vitro drug release studies were carried out using six baskets USP type 2 (paddle) dissolution apparatus. Buffer changing method was used to mimic the GIT environment [14]. In this method $15 \mathrm{mg}$ equivalent weight of pioglitazone $\mathrm{HCl}$ microspheres were taken in dialysis membrane and tied with a paddle using thread. The buffer change method involves the dissolution studies in both acidic (pH 1.2) and basic $(\mathrm{pH}$ 7.4) media. So it was carried out for all the formulations in acidic media for the first $2 \mathrm{~h}$ and basic media for the remaining dissolution studies. The temperature of the dissolution medium was maintained at $37^{\circ} \mathrm{C} \pm 0.5^{\circ} \mathrm{C}$ at a rotating speed of $100 \mathrm{rpm}$. A sample of $5 \mathrm{ml}$ solution was withdrawn at predetermined time intervals up to $18 \mathrm{~h}$, and passed through a $5 \mu \mathrm{m}$ membrane filter and diluted to a suitable concentration with same dissolution media. Absorbance of this solution was analyzed using UV spectrophotometer at $269 \mathrm{~nm}$. The withdrawn volume was replaced with an equal volume of fresh media. The cumulative percentage drug release was calculated using standard calibration curve. The drug release experiments were conducted in triplicate $(n=3)$.

\subsection{Mechanism of Drug Release}

The mechanism of drug release in the matrix system was reported by the diffusion mechanism. Polymer molecular weight can affect polymer degradation and drug release rates. Microsphere size will strongly affect the rate of drug release. As size decreases, the surface area-to-volume ratio of the particle increases. Thus, for a given rate of drug diffusion through the microsphere, the rate of flux of drug out of the microsphere, per mass of formulation, will increase with decreasing particle size [15]. In addition, a major mechanism for the release of many drugs is diffusion through water-filled pores, formed as polymer degradation generates soluble monomers and oligomers that can diffuse out of the particle. For analyzing the mechanism for the release rate kinetics of the mucoadhesive microspheres, the results of in-vitro release profiles obtained for all the best formulations were fitted into any one of the four models as follows:

1) Cumulative percent drug released versus time (Zero-order kinetic model);

2) Log cumulative percent drug remaining versus time (First-order kinetic model);

3) Cumulative percent drug released versus square root of time (Higuchi's model);

4) Log cumulative percent drug released versus log time (Korsmeyer-peppas equation).

In this by comparing the $\mathrm{R}^{2}$ values obtained, the best-fit model was selected.

\subsection{Zero Order Kinetics}

Drug dissolution from Pharmaceutical dosage forms that do not disaggregate and release the drug slowly, assuming that the area does not change and no equilibrium conditions are obtained can be represented by the fol- 
lowing equation.

$$
Q_{t}=Q_{0}+K_{0} t
$$

where, $Q_{t}=$ amount of drug dissolved in time $t$;

$Q_{0}=$ initial amount of drug in the solution;

$K_{0}=$ Zero order release constant.

\subsection{First Order Kinetics}

To study the first order release rate kinetics the release rate data were fitted to the following equation.

$$
\log Q_{t}=\log Q_{0}+K_{1} t / 2.303
$$

where, $Q_{t}=$ amount of drug released in time $t$;

$Q_{0}=$ initial amount of drug in the solution;

$K_{1}=$ first order release rate constant.

\subsection{Higuchi Model}

Higuchi developed several theoretical models to study the release of water soluble and low soluble drugs in corporate in semisolids and or solid matrices.

Mathematical expressions were obtained for drug particles dispersed in a uniform matrix behaving as the diffusion media.

$$
Q_{t}=K_{H} \cdot t_{1 / 2}
$$

where, $Q_{t}=$ amount of drug released in time $t$;

$$
K_{H}=\text { Higuchi dissolution constant. }
$$

\subsection{Korsmeyer and Peppas Release Model}

To study this model the release rate data are fitted to the following equation.

$$
M_{t} / M_{\infty}=K \cdot t^{n}
$$

where, $M_{t} / M_{\infty}=$ fraction of drug release;

$K=$ release constant;

$t=$ release time;

$n=$ diffusional exponent indicative of the mechanism drug release.

If $n$ value is 0.5 or less, the release mechanism follows "Fickian diffusion" and higher values of $0.5<n<1$ for mass transfer follow a non-fickian model (anomalous transport). The drug release follows zero-order drug release and case-II transport if the $n$ value is 1 . For the values of $n$ higher than 1 , the mechanism of drug release is regarded as super case-II transport. This model is used to analyse the release of pharmaceutical polymeric dosage forms when the release mechanism is not known or more than one type of release phenomenon was involved. The $n$ value could be obtained from slope of the plot of log cumulative $\%$ of drug released vs $\log$ time.

\subsection{Stability Studies}

The stability of a drug product is the ability of a particular formulation, in a specific container, to remain within its physico-chemical, therapeutic and toxicological specifications. Stability testing provides evidence on how the quality of a drug substance or drug product varies with time under the influence of a variety of environmental factors such as temperature, humidity, and light and enables recommended storage conditions, retest periods, and shelf lives to be established [16]. Pioglitazone microspheres were filled in HDPE containers at $40^{\circ} \mathrm{C} \pm 2^{\circ} \mathrm{C} /$ $75 \% \pm 5 \% \mathrm{RH}$ for 3 months as per ICH specifies the length of study and storage conditions [17]. The samples were then characterized for percentage of drug content [18].

\section{Results and Discussion}

To develop a stable formulation, the drug-excipient interaction study is performed during the product develop- 
ment stage generally employing FTIR spectroscopy. Lack of new characteristic peaks in the FTIR spectra indicates the absence of drug-excipient interactions. Figure 1(a) shows the FTIR spectra of pure pioglitazone. Figure 1(b) shows the FTIR spectra of Pioglitazone + Sodium-CMC microspheres. Figure 1(c) shows the FTIR spectra of Pioglitazone + Carbopol 940 miscrospheres. Figure 1(d) shows the FTIR spectra of Pioglitazone + Cellulose acetate butratemiscrospheres.

The different formulations were done by using sodium alginate as a core polymer in combination with four mucoadhesive polymers namely sodium carboxy methyl cellulose, carbopol $934 \mathrm{P}$, hydroxy propyl methyl cellulose, and cellulose acetate phthalate as coat materials. The pioglitazone is practically water insoluble and its absorption is dissolution rate limited. The pure drug of pioglitazone $\mathrm{HCl}$ and the solid admixture of the drug and various ratios of polymer were used in the preparation of mucoadhesive microspheres were characterized by FTIR spectroscopy and DSC to know the compatibility. As shown in Figure 1, there was no significant difference or the characteristic peak of pure drug was unchanged in the spectrum of different formulations. The DSC thermo gram of pure pioglitazone and drug loaded microspheres were showed that there was absence of drug and polymer interaction (Figure 2). The particle size of microspheres was found to be between $530 \pm 1.26 \mu \mathrm{m}$ and $793 \pm 1.62 \mu \mathrm{m}$ (Table 3). The SEM analysis results showed the microspheres to be discrete and spherical (Figure 3). The SEM photographs indicated that microspheres were completely covered the coat polymer. Figure 3(a) shows Pioglitazone $\mathrm{HCl}$ loaded group of microspheres at $\times 35$ magnification. Figure 3(b) shows Pioglitazone $\mathrm{HCl}$ loaded single microspheres at $\times 160$ magnification. The ex-vivo mucoadhesive study was performed by in vivo wash off test method. The mucoadhesive strength of prepared microspheres were tested in presence of simulated gastric fluid (SGF, $\mathrm{pH} 1.20 .1 \mathrm{M} \mathrm{HCl}$ ) and simulated intestinal fluid (SIF, pH 7.4 phosphate buffer) and the results are summarized in Figure 4 and Figure 5. The mucoadhesive strength of microspheres was directly proportional to the polymer concentration in both the media. Although the maximum value of mucoadhesive strength was attained by the combination of polymers, significant mucoadhesive strengths were also shown by individual polymers in both simulated gastric and simulated intestinal fluids. The optimized formulation PMI 8 showed considerable mucoadhesive strength with a mucoadhesive time over more than $7 \mathrm{~h}$.

In the first $2 \mathrm{~h}, 1.2 \mathrm{pH}$ acidic media, the drug release of microspheres was low (less than 15\%). This indicates

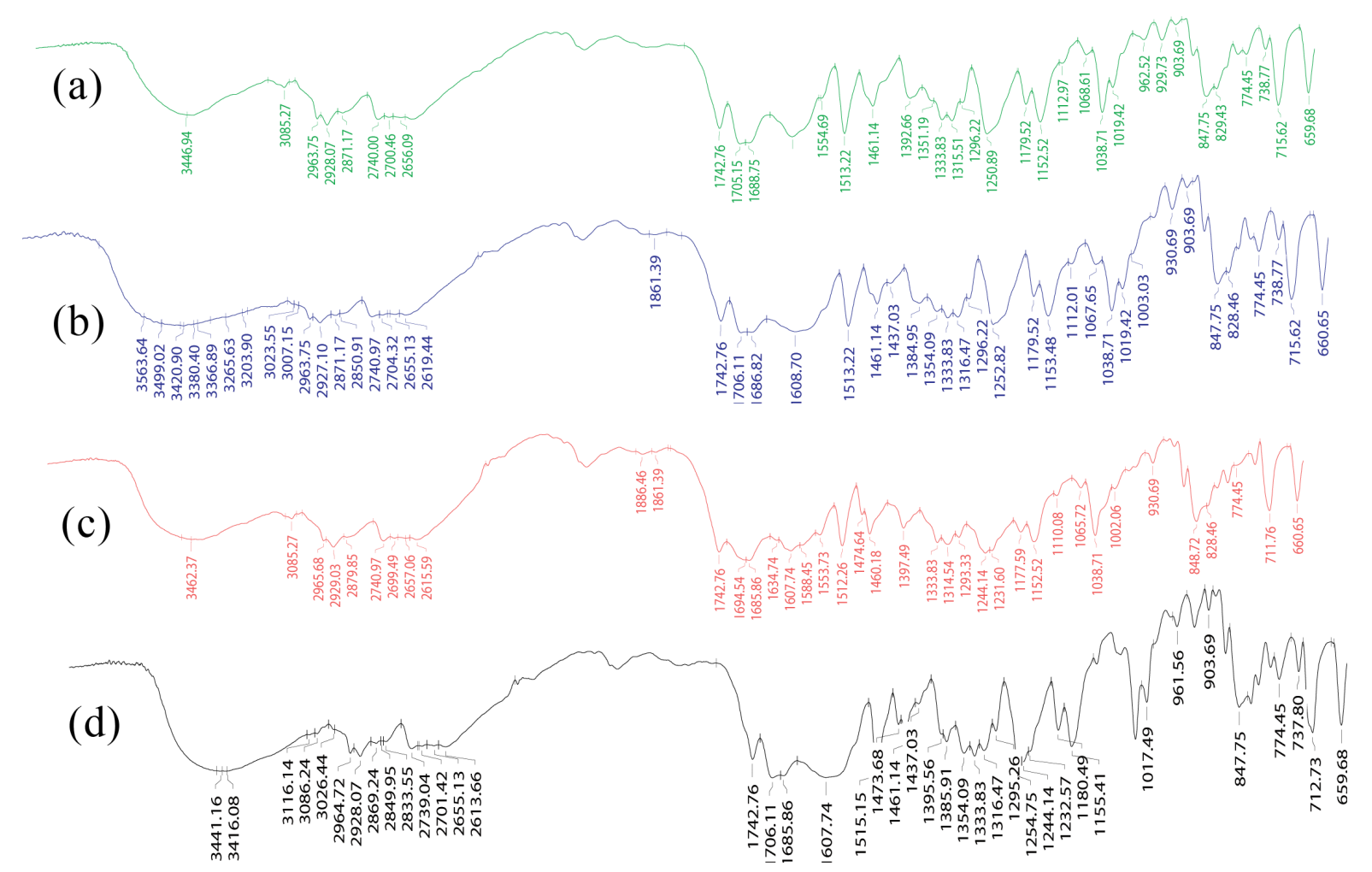

Figure 1. FTIR Spectra of (a) Pure pioglitazone; (b) Pioglitazone + Sodium CMC microspheres; (c) Pioglitazone + Carbopol 940 miscrospheres; (d) Pioglitazone + Cellulose Acetate Butrate miscrospheres. 


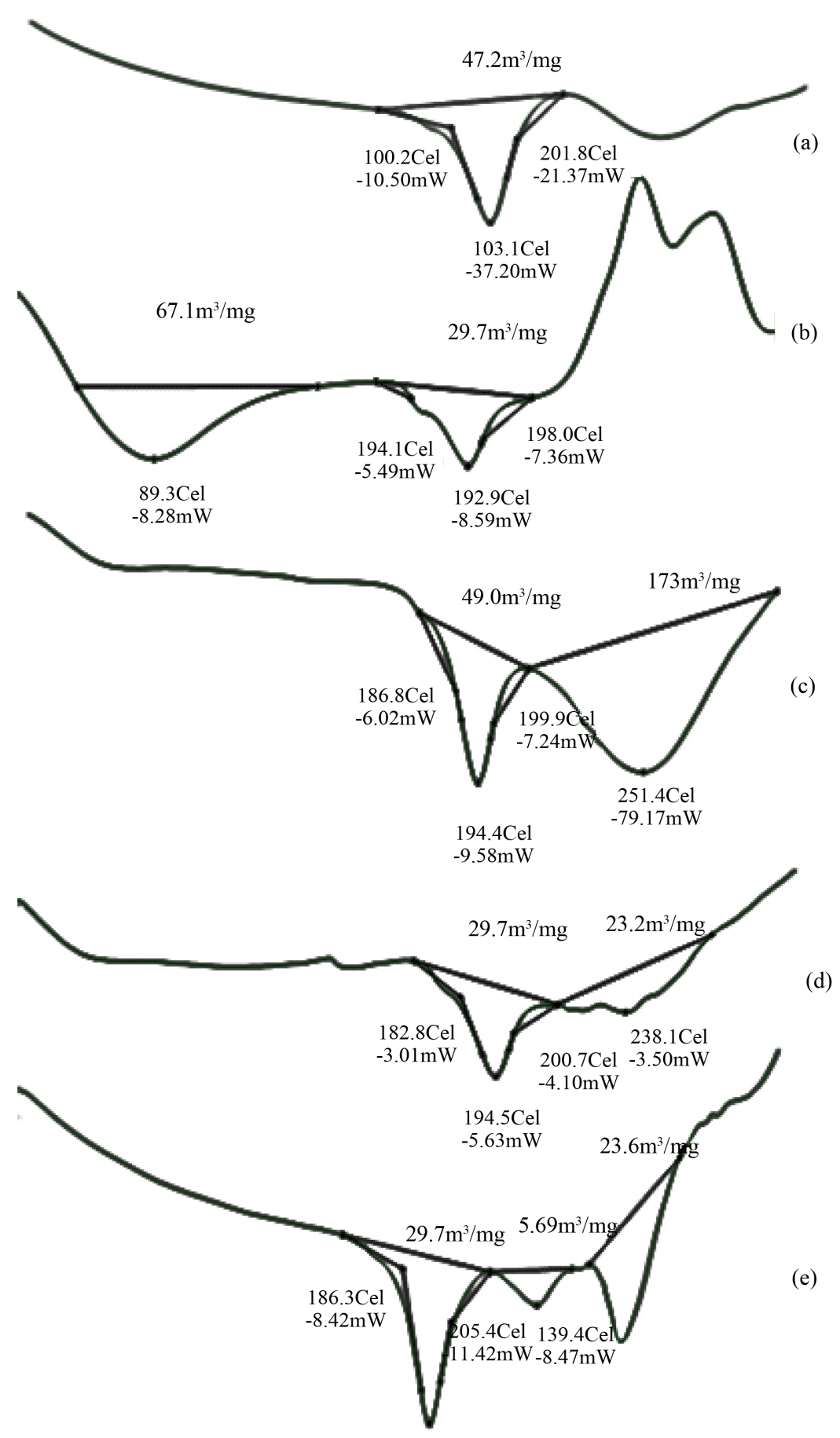

Figure 2. DSC thermograms of (a) Pure pioglitazone; (b) Pioglitazone loaded Sodium alginate microspheres; (c) Pioglitazone loaded Carbopol 934 microspheres; (d) Pioglitazone loaded HPMC microspheres; (e) Pioglitazone loaded CAB microspheres.

that the microspheres did not release the more drug in the acidic media. After changing into basic media, we can get the sustained release of the drug. It indicates, the microspheres released less in acidic $\mathrm{pH}$, and also swelling of microspheres in the acidic media was low. However the basic $\mathrm{pH}$ of the media was able to penetrate the microspheres and dissolve the matrix to release the drug by diffusion. And also observed, in all formulations, with the increase in the polymer concentration, the rate and amount of drug release was decreased, which can be 


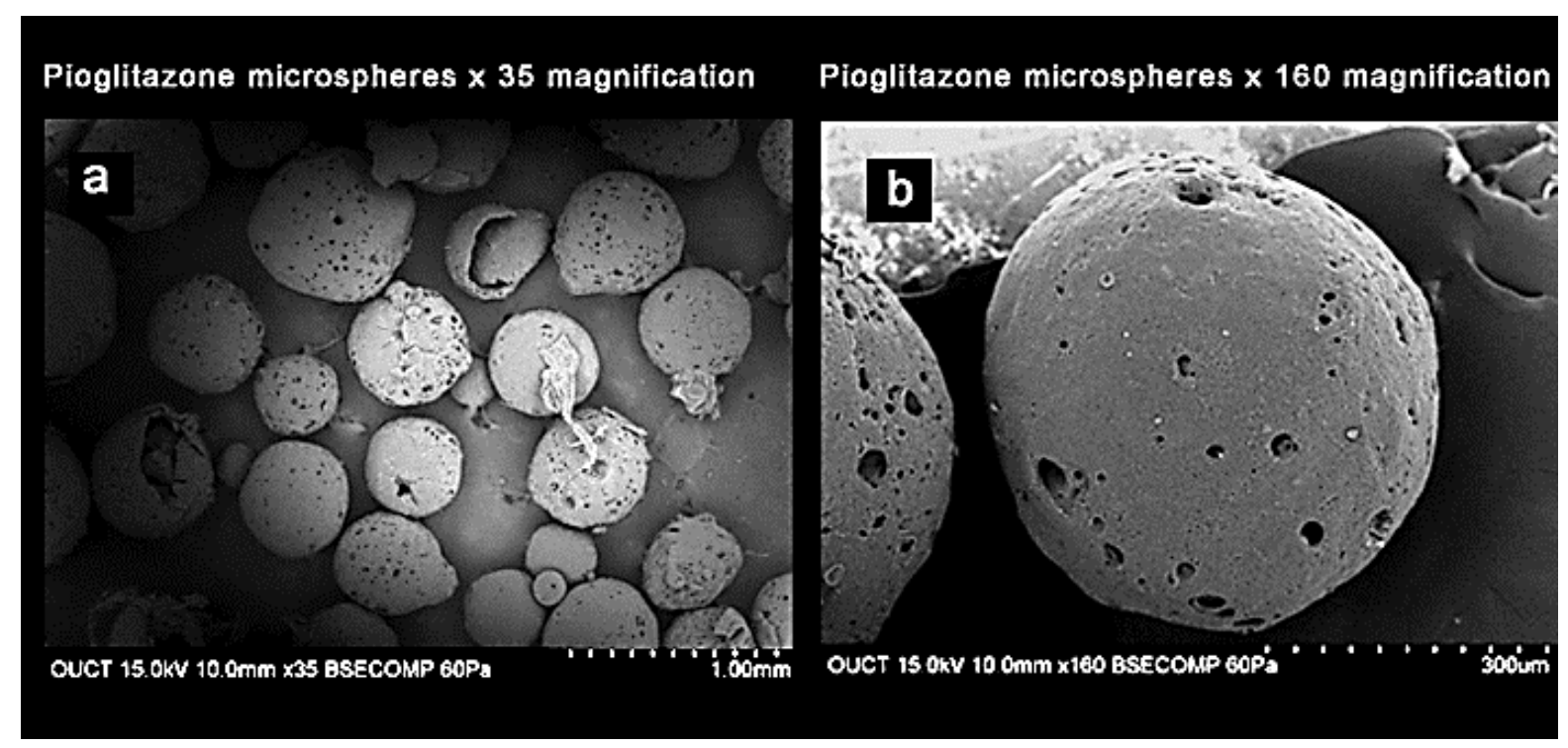

Figure 3. (a) Pioglitazone $\mathrm{HCl}$ loaded group of microspheres at $\times 35$ magnification; (b) Pioglitazone $\mathrm{HCl}$ loaded single microspheres at $\times 160$ magnification.

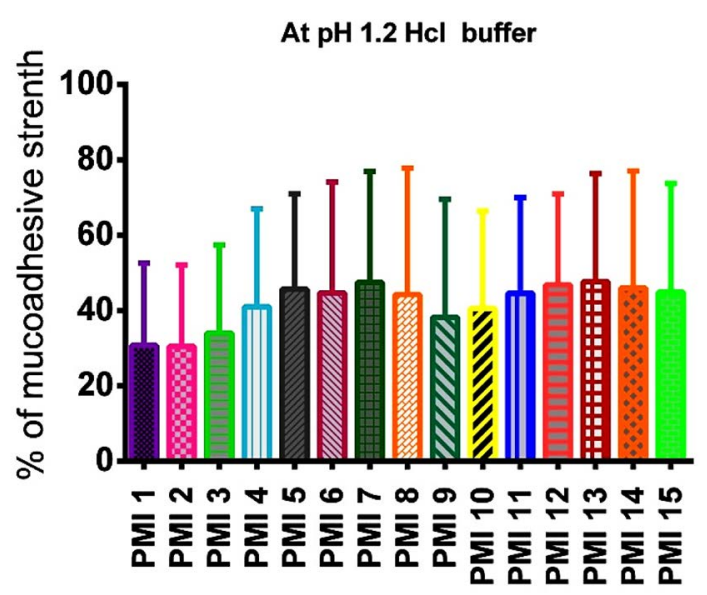

Figure 4. Mucoadhesive strength of various formulations at $\mathrm{pH} 1.2 \mathrm{Hcl}$ buffer.

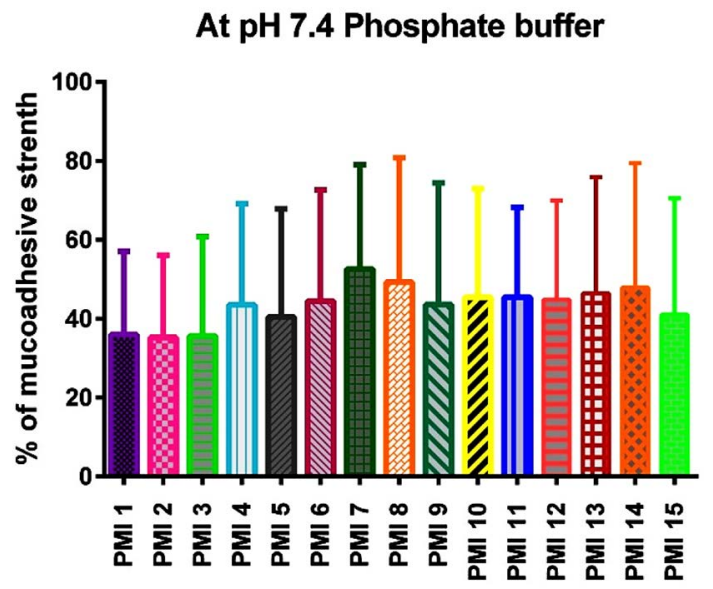

Figure 5. Mucoadhesive strength of various formulations at pH 7.4 phosphate buffer scanning electron microspheres. 
Table 3. Characterization of drug encapsulated microspheres.

\begin{tabular}{cccc}
\hline Formulation & Particle size $(\mu \mathrm{m})$ & Drug entrapment (\%) & Swelling index (\%) \\
PMI 1 & $530 \pm 1.26$ & $56.12 \pm 3.86$ & $76 \pm 3.17$ \\
PMI 2 & $572 \pm 02.38$ & $58.28 \pm 2.32$ & $79 \pm 2.08$ \\
PMI 3 & $594 \pm 1.78$ & $60.58 \pm 1.72$ & $86 \pm 1.52$ \\
PMI 4 & $618 \pm 1.24$ & $68.72 \pm 2.87$ & $78 \pm 2.64$ \\
PMI 5 & $638 \pm 1.78$ & $71.08 \pm 4.22$ & $80 \pm 1.45$ \\
PMI 6 & $762 \pm 2.04$ & $72.66 \pm 3.85$ & $87 \pm 0.98$ \\
PMI 7 & $625 \pm 1.38$ & $72.82 \pm 1.73$ & $70 \pm 1.65$ \\
PMI 8 & $652 \pm 0.88$ & $84.68 \pm 2.93$ & $75 \pm 2.65$ \\
PMI 9 & $793 \pm 1.62$ & $78.52 \pm 1.78$ & $78 \pm 1.29$ \\
PMI 10 & $542 \pm 1.78$ & $74.34 \pm 2.73$ & $68 \pm 1.52$ \\
PMI 11 & $556 \pm 2.04$ & $69.73 \pm 2.75$ & $72 \pm 3.85$ \\
PMI 12 & $609 \pm 1.38$ & $72.87 \pm 2.88$ & $74 \pm 3.74$ \\
PMI 13 & $547 \pm 0.88$ & $70.92 \pm 3.58$ & $74 \pm 4.12$ \\
PMI 14 & $561 \pm 1.62$ & $71.09 \pm 1.98$ & $79 \pm 2.65$ \\
PMI 15 & $576 \pm 0.88$ & $66.24 \pm 3.72$ & $83 \pm 3.73$ \\
\hline
\end{tabular}

Mean \pm Standard deviation $(\mathrm{n}=3)$

attributed to the greater binding of the drug with polymer. This is due to large amount of drug got bounded in the polymer matrix, as a result the rate of drug release from the microspheres was decreased. The in vitro release of pioglitazone hydrochloride was influenced by altering the drug to polymer ratio and the mechanism of drug release was by diffusion controlled. The percentage drug release of each of the formulation was found to be PMI 1 (60.23\%), PMI 2 (57.26\%), PMI 3 (51.26\%), PMI 4 (70.69\%), PMI 5 (72.83\%), PMI 6 (75.49\%), PMI 7 (74.38\%), PMI 8 (82.12\%), PMI 9 (76.54\%), PMI 10 (74.94\%), PMI 11 (66.08\%), PMI 12 (69.45\%), PMI 13 (78.36\%), PMI 14 (73.54\%), PMI 15 (67.52\%) of drug release at the end of $18 \mathrm{~h}$ (Figures 6(a)-(e)). So it is the best formulation releasing the drug at a controlled rate for a period of $18 \mathrm{~h}$ increase drug absorption further increasing the bioavailability.

\subsection{Mechanism of Drug Release}

The matrix system was reported the diffusion mechanism for the release of the drug. The dissolution kinetic data of pioglitazone microspheres are summarized (Table 4). As observed from the various plots, most of the formulations followed the Korsmeyer-peppasmodel. The optimized formulation PMI 8 followed the Korsmeyer-peppas model. Based on the "n" value it followed non-fickian transport mechanism. Non-fickian transport mechanism refers to a combination of both diffusion and erosion controlled drug release. This indicates that the prepared formulations are suitable as sustained release dosage forms.

\subsection{Stability Studies}

The stability studies were carried out for the formulation PMI 8 at $40^{\circ} \mathrm{C} \pm 2{ }^{\circ} \mathrm{C} / 75 \% \pm 5 \%$ RH for 3 months as per ICH guidelines and the results are summarized in Table 5. The results indicated that the microspheres did not show any significant physical changes during the study period. The results of stability studies show that there is about $84.65 \pm 2.93$ percentage of the drug is present in the formulation and $82.06 \pm 1.84$ percentage of in vitro drug release after storage at $45^{\circ} \mathrm{C}$ for 90 days, it indicates the good stability of the pioglitazone microspheres. 


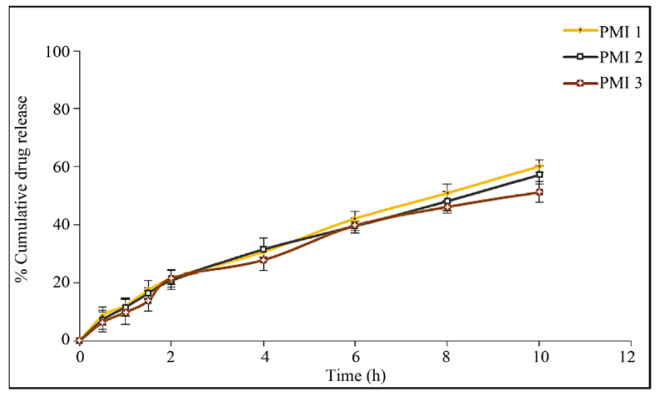

(a)

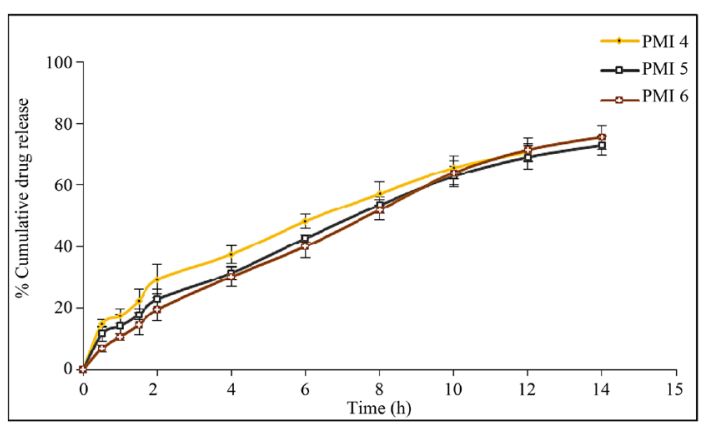

(b)

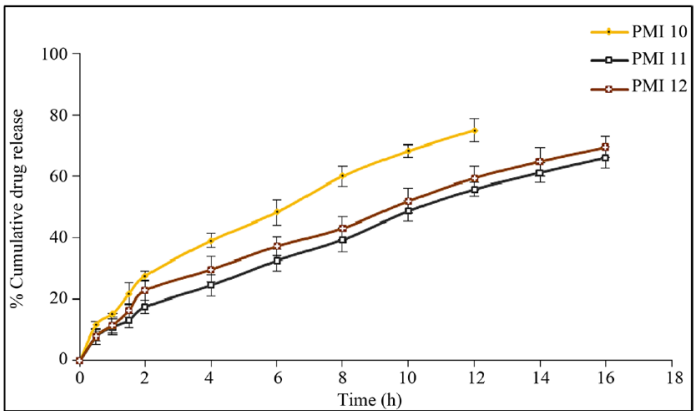

(d)

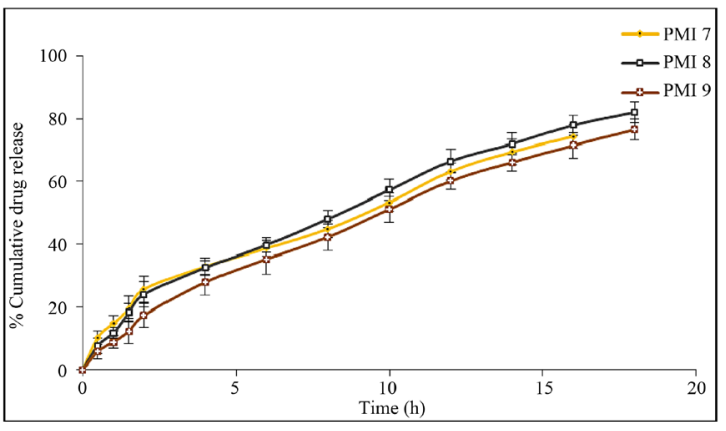

(c)

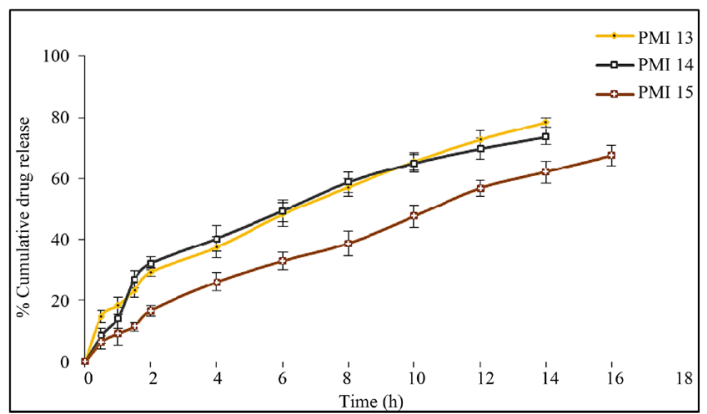

(e)

Figure 6. (a) In vitro release data of pioglitazone $\mathrm{HCl}$ microspheres PMI 1 to PMI 3; (b) In vitro release data of pioglitazone $\mathrm{HCl}$ microspheresPMI4 to PMI6; (c) In vitro release data of pioglitazone $\mathrm{HCl}$ microspheres PMI 7 to PMI 9; (d) In vitro release data of pioglitazone $\mathrm{HCl}$ microspheres PMI 10 to PMI 12; (e) In vitro release data of pioglitazone $\mathrm{HCl}$ microspheres PMI 13 to PMI 15.

\section{Mucoadhesive Studies}

The in vitro mucoadhesive study was performed by in vitro wash off test method. The mucoadhesive strength of prepared microspheres was tested in the presence of simulated gastric fluid (SGF, pH 1.2 0.1M HCl) and simulated intestinal fluid (SIF, pH 7.4 phosphate buffer) and the results are summarized in Figure 4 and Figure 5. The mucoadhesive strength of microspheres was directly proportional to the polymer concentration in both the media. Although the maximum value of mucoadhesive strength was attained by the combination of polymers, significant mucoadhesive strengths were also shown by individual polymers in both simulated gastric and simulated intestinal fluids. The optimized formulation PMI 8 showed considerable mucoadhesive strength with a mucoadhesive time over more than $7 \mathrm{~h}$.

\section{Conclusions}

In the present study, mucoadhesive microspheres of pioglitazone $\mathrm{HCl}$ were prepared successfully using ionotropic external gelation method. Ratio of drug: polymer and stirring speed of the mechanical stirrer was important to obtain spherical particles with smooth surfaces. The yields of formulated microspheres and encapsulation 
Table 4. Release kinetic data of formulations PMI-1-PMI-15.

\begin{tabular}{|c|c|c|c|c|c|c|c|c|c|c|}
\hline \multirow{2}{*}{ Formulation } & \multirow{2}{*}{$\mathbf{T}_{50}(\mathrm{~h})$} & \multicolumn{2}{|c|}{ Zero-order } & \multicolumn{2}{|c|}{ First-order } & \multicolumn{2}{|c|}{ Higuchi } & \multicolumn{2}{|c|}{$\begin{array}{c}\text { Korsmeyer } \\
\text { Peppas }\end{array}$} & \multirow{2}{*}{ Best fit } \\
\hline & & $\mathbf{R}^{2}$ & $\begin{array}{c}\mathbf{K}_{0} \\
\left(\mathbf{m g} \cdot \mathbf{h r} \mathbf{r}^{-1}\right)\end{array}$ & $\mathbf{R}^{2}$ & $\begin{array}{c}\mathbf{K}_{1} \\
\left(\mathbf{h r}^{-1}\right)\end{array}$ & $\mathbf{R}^{2}$ & $\mathbf{K}_{\mathbf{H}}$ & $\mathbf{R}^{2}$ & $\mathbf{N}$ & \\
\hline PMI 1 & 4 & 0.9770 & 5.626 & 0.9944 & 0.038 & 0.9825 & 19.35 & 0.9964 & 0.650 & $\mathrm{a}$ \\
\hline PMI 2 & 4 & 0.9720 & 5.359 & 0.9932 & 0.035 & 0.9853 & 18.51 & 0.9967 & 0.678 & $\mathrm{a}$ \\
\hline PMI 3 & 4 & 0.9580 & 5.012 & 0.9884 & 0.031 & 0.9797 & 17.39 & 0.9848 & 0.702 & $\mathrm{c}$ \\
\hline PMI 4 & 4 & 0.9476 & 5.355 & 0.9909 & 0.042 & 0.9954 & 20.61 & 0.9878 & 0.517 & $\mathrm{~b}$ \\
\hline PMI 5 & 4 & 0.9707 & 5.016 & 0.9954 & 0.040 & 0.9866 & 20.50 & 0.9885 & 0.5906 & $\mathrm{c}$ \\
\hline PMI 6 & 4 & 0.9841 & 5.427 & 0.9929 & 0.044 & 0.9749 & 21.89 & 0.9979 & 0.737 & $\mathrm{a}$ \\
\hline PMI 7 & 4 & 0.9656 & 4.231 & 0.9851 & 0.034 & 0.9876 & 18.53 & 0.9916 & 0.557 & $\mathrm{a}$ \\
\hline PMI 8 & 4 & 0.9704 & 4.393 & 0.9918 & 0.039 & 0.9897 & 20.37 & 0.9919 & 0.651 & $\mathrm{a}$ \\
\hline PMI 9 & 4 & 0.9822 & 4.200 & 0.9948 & 0.077 & 0.9829 & 19.29 & 0.9974 & 0.734 & $\mathrm{a}$ \\
\hline PMI 10 & 4 & 0.9619 & 5.855 & 0.9952 & 0.047 & 0.9928 & 22.34 & 0.9947 & 0.602 & $\mathrm{c}$ \\
\hline PMI 11 & 4 & 0.9842 & 3.958 & 0.9956 & 0.065 & 0.9813 & 17.11 & 0.9948 & 0.637 & $\mathrm{c}$ \\
\hline PMI 12 & 4 & 0.9680 & 4.088 & 0.9935 & 0.070 & 0.9902 & 17.90 & 0.9922 & 0.621 & $\mathrm{c}$ \\
\hline PMI 13 & 4 & 0.9543 & 5.067 & 0.9929 & 0.044 & 0.9963 & 20.99 & 0.9933 & 0.514 & $\mathrm{~b}$ \\
\hline PMI 14 & 4 & 0.9103 & 4.919 & 0.9808 & 0.092 & 0.9865 & 20.76 & 0.9521 & 0.616 & $\mathrm{~b}$ \\
\hline PMI 15 & 4 & 0.9856 & 4.098 & 0.9938 & 0.067 & 0.9780 & 17.68 & 0.9956 & 0.703 & $\mathrm{a}$ \\
\hline
\end{tabular}

$\mathrm{R}^{2}=$ correlation coefficient; $\mathrm{K}_{\mathrm{H}}=$ Higuchi constant; $\mathrm{N}=$ Koresmeyer Peppas constant; $\mathrm{a}=$ Peppas; $\mathrm{B}=$ Higuchi; $\mathrm{c}=$ First order; $\mathrm{d}=$ Zero order.

Table 5. Stability data of optimized formulation physico-chemical parameters.

\begin{tabular}{ccccc}
\hline S.No & $\begin{array}{c}\text { Time in } \\
\text { days }\end{array}$ & $\begin{array}{c}\text { Physical } \\
\text { changes }\end{array}$ & $\begin{array}{c}\text { Percentage } \\
\text { drug content (mg) }\end{array}$ & $\begin{array}{c}\text { Percentage of in vitro } \\
\text { drug release }\end{array}$ \\
\hline 1. & 0 & --- & $84.68 \pm 1.09$ & $82.12 \pm 3.24$ \\
2. & 15 & No change & $84.68 \pm 1.12$ & $82.10 \pm 2.48$ \\
3. & 30 & No change & $84.67 \pm 1.26$ & $82.09 \pm 2.23$ \\
4. & 45 & No change & $84.67 \pm 1.78$ & $82.09 \pm 1.52$ \\
5 & 60 & No change & $84.66 \pm 2.93$ & $82.08 \pm 1.36$ \\
6 & 75 & No change & $84.65 \pm 2.93$ & $82.08 \pm 3.44$ \\
7 & 90 & No change & $84.65 \pm 2.93$ & $82.06 \pm 1.84$ \\
\hline
\end{tabular}

Mean \pm standard deviation $(\mathrm{n}=3)$.

efficiencies were high in most of the prepared microspheres. The practical yield of prepared microspheres using the ionotropic gelation technique was between $172 \mathrm{mg}$ and $604 \mathrm{mg}$. The result of the Chi-squared test carried out between the actual (practical) and expected (theoretical) yields showed no significant difference $(\mathrm{P}<0.05)$ which indicated that the ionotropic gelation technique could be successfully employed to prepare pioglitazone microspheres using sodium alginate, sodium carboxy methyl cellulose, carbopol 934 P, HPMC, cellulose acetate butyrate polymers.

The drug entrapment efficiency of prepared microspheres showed between $56.12 \% \pm 3.86 \%$ to $84.68 \% \pm$ $2.93 \%$ which is significantly higher for ionotropic gelation technique. Highest drug entrapment was found informulation PMI 8. Swelling index is the capability of a polymer to swell before the drug is released which influences the rate and mechanism of drug release from the polymer matrix. The swelling index of prepared microspheres was in the range of $68 \% \pm 4.52 \%$ to $87 \% \pm 0.98 \%$. Pioglitazone $\mathrm{HCl}$ microspheres showed controlled release of drug without initial peak level achieving. This type of properties in Pioglitazone $\mathrm{HCl}$ microspheres used to decrease side effects, reduce dosing frequency and improve patient compliances. From the all 
batches PMI 8 is considered the best formulation, because among all other formulations, it shows better extent of drug release up to $82.12 \%(18 \mathrm{~h})$, good entrapment efficiency $(84.68 \%)$ and the ex-vivo wash-off test shows the best mucoadhesive property.

\section{References}

[1] Liu, Z., Lu, W., Qian, L., Zhang, X., Zeng, P. and Pan, J. (2005) In Vitro and in Vivo Studies on Mucoadhesive Microspheres of Amoxicillin. Journal of Controlled Release, 102, 135-144. http://dx.doi.org/10.1016/j.jconrel.2004.06.022

[2] Wattamwar, M., Ratnaparkhi, M.P., Kutmalge, M.D. and Jadhav, A.N. (2014) Formulation and in Vitro Evaluation of Mucoadhesive Microspheres of Pioglitazone Hydrochloride. Asian Pacific Journal of Health Sciences, 1, 177-192.

[3] Thompson, C.J., Hansford, D., Higgins, S., Rostron, C., Hutcheon, G.A. and Munday, D.L. (2007) Evaluation of Ibuprofen-Loaded Microspheres Prepared from Novel Copolyesters. International Journal of Pharmaceutics, 329, 53-61. http://dx.doi.org/10.1016/j.ijpharm.2006.08.019

[4] Thanoo, B.C., Sunny, M.C. and Jayakrishnan, A. (1992) Cross-Linked Chitosan Microspheres: Preparation and Evaluation as a Matrix for the Controlled Release of Pharmaceuticals. Journal of Pharmacy and Pharmacology, 44, 283-286. http://dx.doi.org/10.1111/j.2042-7158.1992.tb03607.x

[5] Patil, S., Babbar, A., Mathur, R., Misha, A. and Sawant, K. (2015) Mucoadhesive Chitosan Microspheres of Carvedilol for Nasal Administration. Journal of Drug Targeting, 18, 321-331. http://dx.doi.org/10.3109/10611861003663523

[6] Nadigoti, J., Dharani, S., Shayeda and Yamsani, M.R. (2011) Formulation and Evaluation of Floating Microparticles of Metoprolol Succinate. Asian Journal of Pharmaceutical and Clinical Research, 4, 132-135.

[7] Sabitha, P., Vijayaratna, J. and Reddy, K.R. (2010) Design and Evaluation of Controlled Release Chitosan-Calcium Alginate Microspheres of Anti-Tubercular Drugs for Oral Use. International Journal of Chemtech Research, 2, 88-98.

[8] Mitra, A. and Dey, B. (2011) Chitosonmicropheres in Novel Drug Delivery Systems. Indian Journal of Pharmaceutical Sciences, 73, 355-366.

[9] Xu, J., Bovet, L. and Zhao, K. (2008) Taste Masking Microspheres for Orally Disintegrating Tablets. International Journal of Pharmaceutics, 359, 63-69. http://dx.doi.org/10.1016/j.ijpharm.2008.03.019

[10] Al-Kassas, R.S., Al-Gohary, O.M.N. and Al-Faadhel, M.M. (2007) Controlling of Systemic Absorption of Gliclazide though Incorporation into Alginate Beads. International Journal of Pharmaceutics, 341, 230-237. http://dx.doi.org/10.1016/j.ijpharm.2007.03.047

[11] Gaba, P., Singh, S. and Gaba, M. (2011) Galactomannan Gum Coated Mucoadhesive Microspheres of Glipizide for Treatment of Type 2 Diabetes Mellitus: In Vitro and in Vivo Evaluation. Saudi Pharmaceutical Journal, 19, $143-152$. http://dx.doi.org/10.1016/j.jsps.2011.02.001

[12] Jose, S., Prema, M.T., Chacko, A.J., Thomas, A.C. and Souto, E.B. (2011) Colon Specific Chitosan Microspheres for Chronotherapy of Chronic Stable Angina. Colloids and Surfaces B: Biointerfaces, 83, 277-283. http://dx.doi.org/10.1016/j.colsurfb.2010.11.033

[13] Maravajhala, V., Dasai, N., Sepuri, A. and Joginapalli, S. (2009) Design and Evaluation of Niacin Microspheres. Indian Journal of Pharmaceutical Sciences, 71, 663-669. http://dx.doi.org/10.4103/0250-474X.59549

[14] Gangadhar, B.C., Shyam Sundar, R., Varma, V.K., Sleeva Raju, M. and Sai Kiran, M. (2010) Formulation and Evaluation of Indomethacin Microspheres Using Natural and Synthetic Polymers as Controlled Release Dosage Forms. International Journal of Drug Discovery, 2, 8-16. http://dx.doi.org/10.9735/0975-4423.2.1.8-16

[15] Liggins, R.T. and Burt, H.M. (2001) Paclitaxel Loaded Poly (L-Lactic Acid) Microspheres: Properties of Microspheres Made with Low Molecular Weight Polymers. International Journal of Pharmaceutics, 222, 19-33. http://dx.doi.org/10.1016/s0378-5173(01)00690-1

[16] Shah, P., Rajshree, M. and Rane, Y. (2007) Stability Testing of Pharmaceuticals-A Global Perspective. Journal of Pharmacy Research, 6, 1-9.

[17] Stability Testing Guidelines (2003) Stability Testing of New Drug Substances and Products. ICH-Technical Coordination, EMEA, London.

[18] Prakash, K., Raju, P.N., Shanta, K.K. and Lakshmi, M.N. (2007) Preparation and Characterization of Lamivudine Microspheres Using Various Cellulose Polymers. Tropical Journal of Pharmaceutical Research, 6, 841-847. http://dx.doi.org/10.4314/tjpr.v6i4.14668 